\title{
Neurocutaneous melanosis presented with giant congenital melanocytic nevi and primary intracranial melanoma
}

\section{Shakya $A^{1}$, Huang J'}

${ }^{1}$ Anup Shakya, MD resident; ${ }^{2}$ Jinbai Huang, Professor and Head of Department; Department of Medical Imaging and Nuclear medicine (Radiodiagnosis), Jingzhou First People's Hospital, Affiliated to Yangtze University, Medical College, P.R. China.

\begin{abstract}
Neurocutaneous melanosis is a rare neurocutaneous syndrome defined by the presence of multiple and /or giant congenital cutaneous nevi and melanocytic deposits in the central nervous system with the infiltration of leptomeninges. The major medical concern with giant congenital cutaneous nevi is high risk of developing cutaneous melanoma, leptomeningeal melanoma, and neurocutaneous melanosis. Neurological symptoms usually develop before 2 years of age, but rare in the second or third decades of life. A 24-year-old-man with giant hairy, pigmented nevus on his extremities, back, "bathing trunk" variety presented with onset of neurological symptoms only in second decade of life. Magnetic resonance imaging of brain revealed a large expansive lesion in the left parieto-occipital lobe with high intensity on T1sequence, and was heterogeneous on T2-sequence with iso- and hyper intense areas. Post-surgical pathological diagnosis revealed characteristics of malignant melanoma. Our patient represents a rare association between neurocutaneous melanosis with giant congenital cutaneous nevi and development of primary intracranial melanoma with neurological symptoms appearing in adult life. The onset of neurological symptoms only in third decade of life in our patient illustrates the importance of recognizing the likelihood of neurocutaneous melanosis in children with giant congenital nevus with or without neurological symptoms.
\end{abstract}

Key words: Congenital melanocytic nevi, Neurocutaneous melanosis, Primary intracranial melanoma

\section{INTRODUCTION}

$\mathrm{N}$ eurocutaneous melanosis (NCM) is a congenital, nonhereditary disorder defined by the presence of multiple/or giant congenital melanocytic nevi (GCMN) associated with abnormal melanin deposits in the brain parenchyma and/or leptomeninges documented by magnetic resonance imaging (MRI) or autopsy'. The pathogenesis of this disorder is presumed to involve a developmental error in the morphogenesis of the embryonal neuroectoderm. A giant nevus is seen at birth and preferred sites include the back of the trunk, the "bathing trunk" region, and the scalp, and neck. Most of the patients develop neurological manifestations before two years of age, and the majorities die within three years of presenting neurological symptoms ${ }^{2}$. We report a rare case of neurocutaneous melanosis with giant congenital nevus with manifestations of neurological symptoms only in third decade of life.

Address for correspondence

\section{Dr. Jinbai Huang}

Professor, Department of Medical Imaging and Nuclear medicine (Radiodiagnosis), Jingzhou First People's Hospital, Affiliated to Yangtze University, Medical College, P.R. China.

E-mail: jinbaihuang@126.com

\section{CASE REPORT}

A 24-years-old male presented with one month history of intermittent dizziness, headache, vomiting with disturbance of concentration and progressive forgetfulness prior to the admission in our hospital on $9^{\text {th }}$ February, 2014. The striking feature was the presence of a giant, hairy, pigmented nevus on his extremities, back, more than half of the anterior thoracic region and multiple disseminated congenitalmelanocytic nevi (Figure 1, Figure 2), "bathing trunk" variety. His neuropsychomotor development was normal. No significant family history was elicited at the time of history-taking.

On admission, ophthalmological examination revealed no significant pathology. No evidence of another primary tumor such as mucosal melanoma or metastases was found. It included computed tomography (CT) scan of the abdomen and thorax, a gastroscopy, colonoscopy, and a routine blood analysis including a negative $\$ 100$ tumor marker.

\section{Radiographic Findings}

A brain CT scan showed high-density mass in the left parietal lobe and edema in the adjacent brain tissue 
(Figure 3). The high density area suggested hematoma. MRI of brain revealed a large expansive lesion measuring $42 \times 38 \times 39 \mathrm{~mm}^{3}$ in the left parietal lobe associated with hemorrhagic component showing rounded morphology and relatively well-defined contours. The lesion showed high intensity on T1 sequence (Figure 4), and was heterogeneous on T2 sequence (Figure5) and T2 FLAIR sequence (Figure 6), with iso- and hyper intense areas. Diffusion weighted image (DWI) shows mixed signal intensity.

\section{Operative Findings}

A left-sided posterolateral approach was performed to expose the lesion. Tumor was situated in the left parieto-occipital region. Base of the tumor was in close relation with the dura mater, purple in color, soft with abundant blood supply, incompletely wrapped in the membrane with clear boundaries with brain tissue, clear old hemorrhage found inside and around the tumor. The tumor was resected completely.

\section{Histopathological Findings}

Microscopy sections of the cutaneous nevi taken from several sites showed the histological appearance of pigmented intradermal nevi. There was no microscopic evidence of malignant change in any of the sections taken. The left parieto-occipital tumor showed the characteristic appearance of malignant melanoma.

\section{Immunohistochemistry}

GFAP (-), S-100 (+), NSE (+), HMB45 (+), MeLan-A (+), CD (-), Ki67 (+): 30\%-40\% (Fig.8, Fig.9)

\section{Treatment}

Surgical removal of brain lesion was followed by chemotherapy. After 10 days of treatment, his headache and dizziness were subsided. He was adviced for the follow up after 3 weeks from the date of discharge. Patient's condition deteriorated few days after his discharge with the onset of severe headache and decreased consciousness and coma. He died 2 months after the initial presentation.

\section{DISCUSSION}

NCM is a rare congenital syndrome characterized by the proliferation of melanin-producing cells in both the skin and the leptomeninges. The great majority of the symptomatic cases become evident by the age of two to three years ${ }^{3}$. Although the exact pathogenesis is obscure, NCM is generally believed to represent an embryonal neuroectodermal dysplasia4. GCMN is, generally defined as a congenital melanocytic lesion that will reach, at least, $20 \mathrm{~cm}$ in the skin in adult life. Despite its rarity, GCMN is important for its association with severe complications such as malignant melanoma

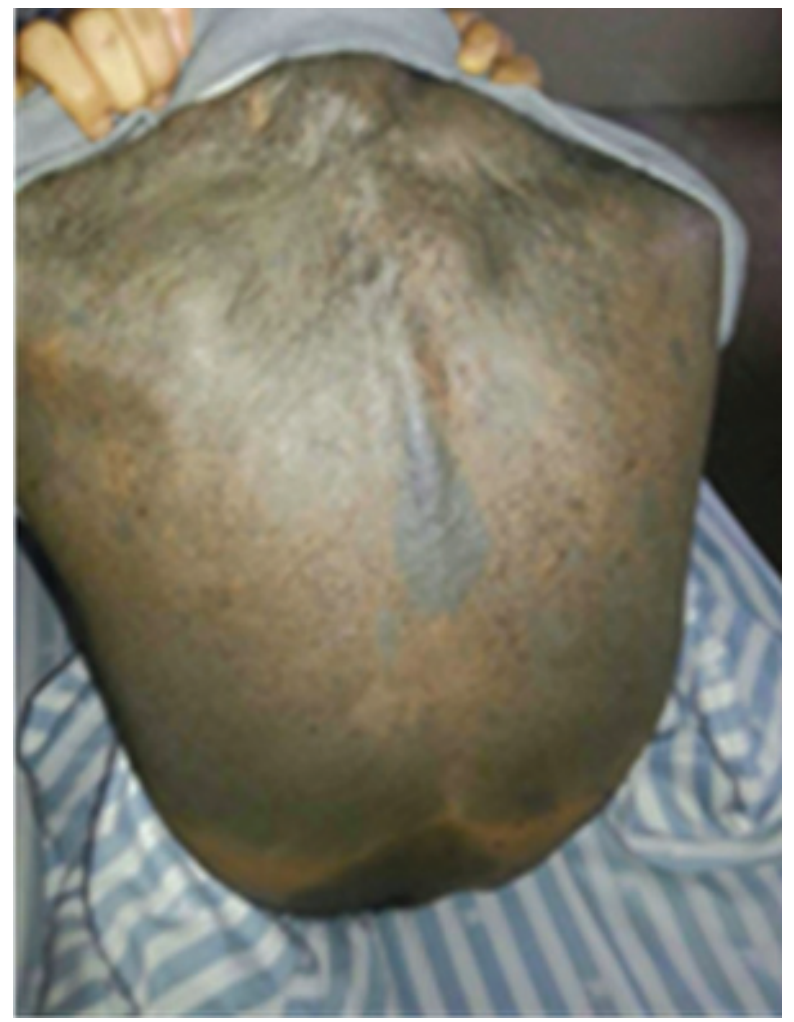

Figure 1: Extensive pigmented patch involving whole back.

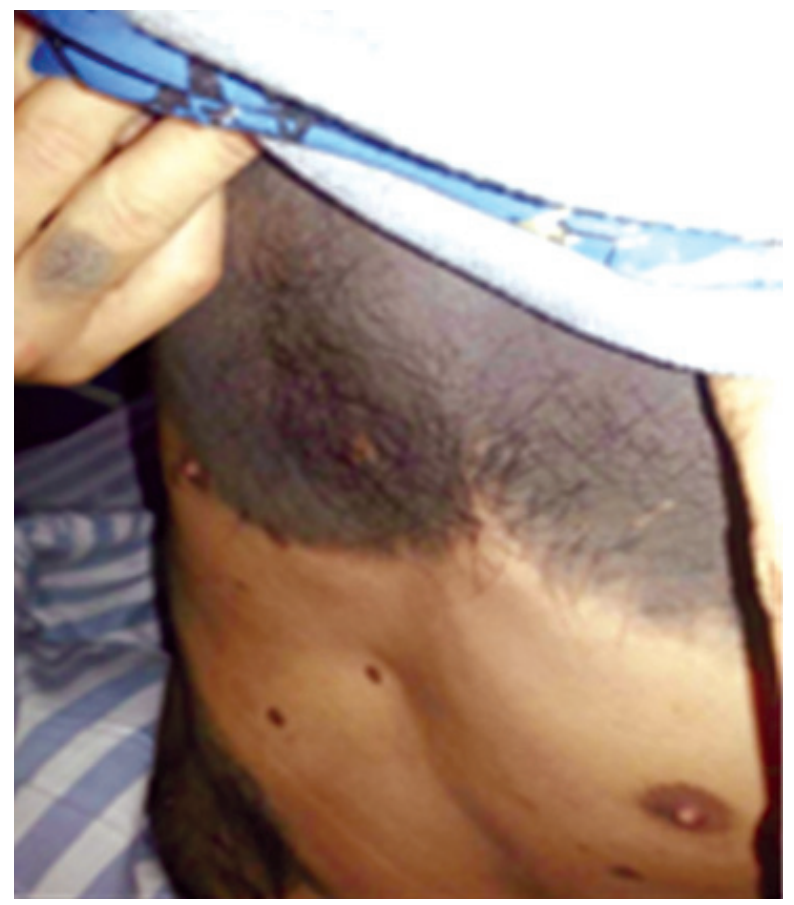

Figure 2: Extensive pigmented patch involving upper part of chest and multiple satellite nevi. 
and central nervous system (CNS) involvement, as well as a major psychosocial impact on the patient and his family, due to its unsightly appearance 5 . Cutaneous lesions are giant and multiple pigmented nevi. The sites of predilection are the back of the trunk, scalp, neck, and the swimming trunks area, which is why it is often called

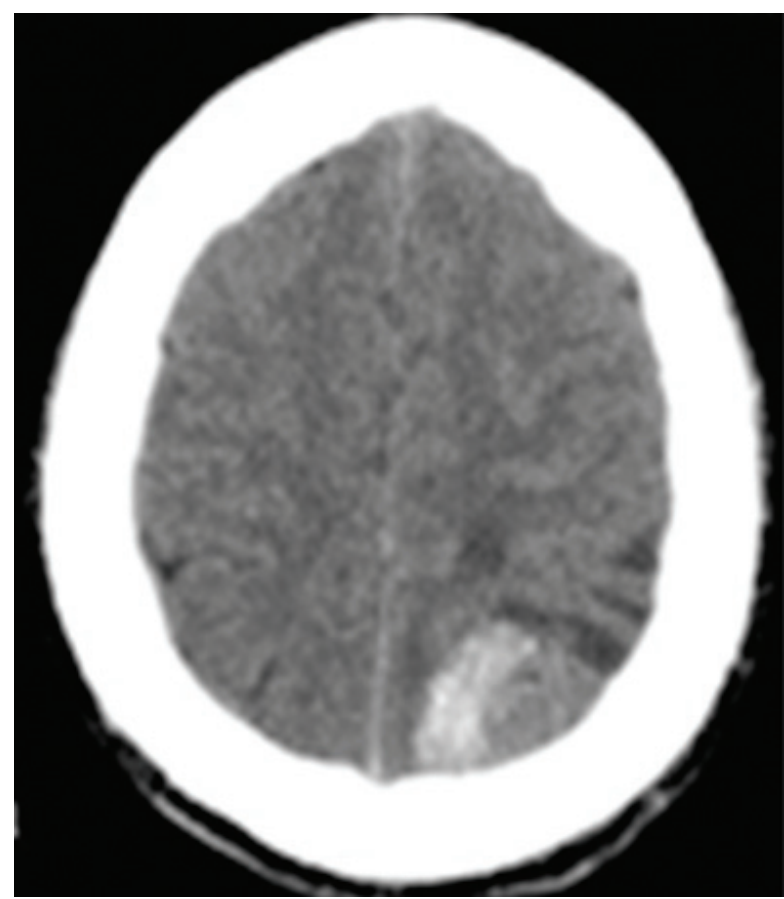

Figure 3: Axial CT shows slightly high density mass with edema

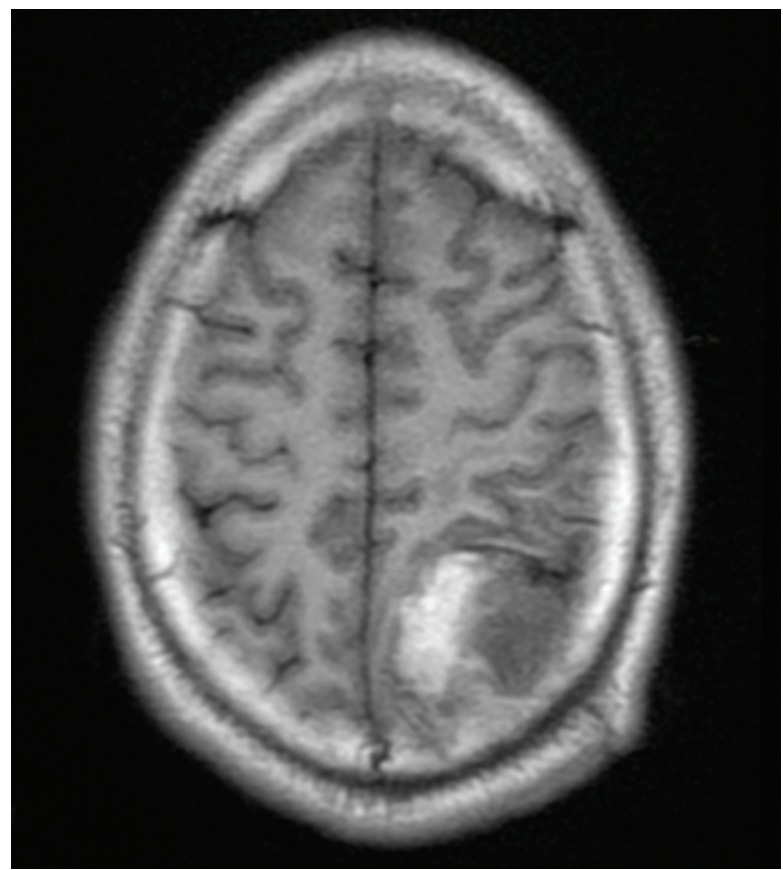

Figure 4: In MRI, T1FLAIR shows mixed signal intensity in left parieto-occipital lobe the left parieto-occipital lobe. "bathing trunk". Frequently these lesions appear on the lumbosacral, occipital, and upper back area. The surfaces of nevi are irregular and often covered with hair, and they often have nearby satellite lesions or are associated with a giant nevus and numerous small nevi scattered over the entire body ${ }^{1}$. Our patient had dark nevi over the

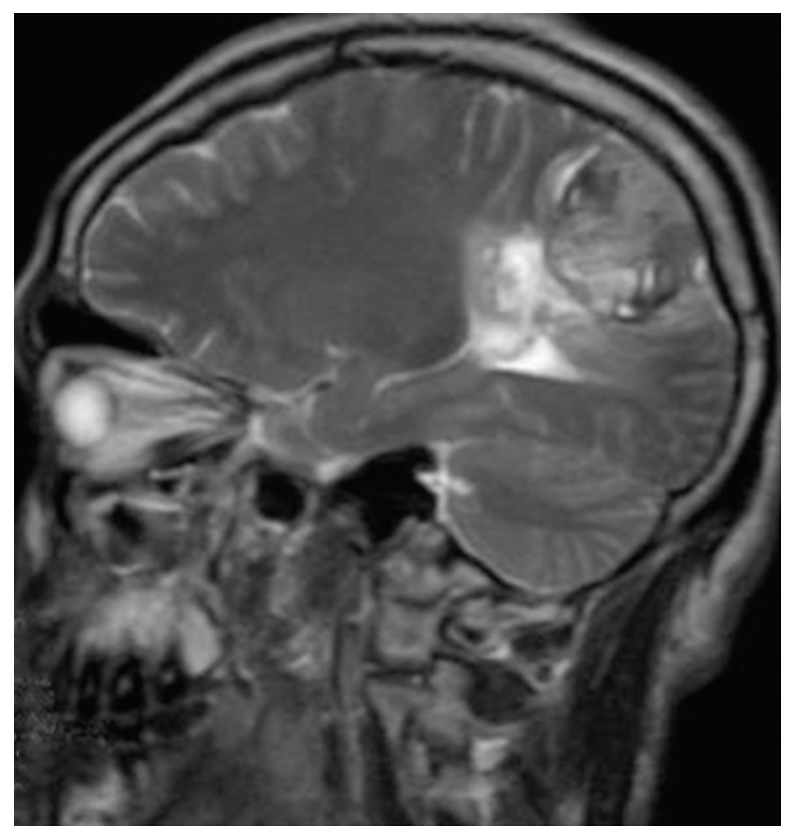

Figure 5: Unenhanced T2-weighted sagittal image documenting the mass at the level of the leftparietooccipital convexity.

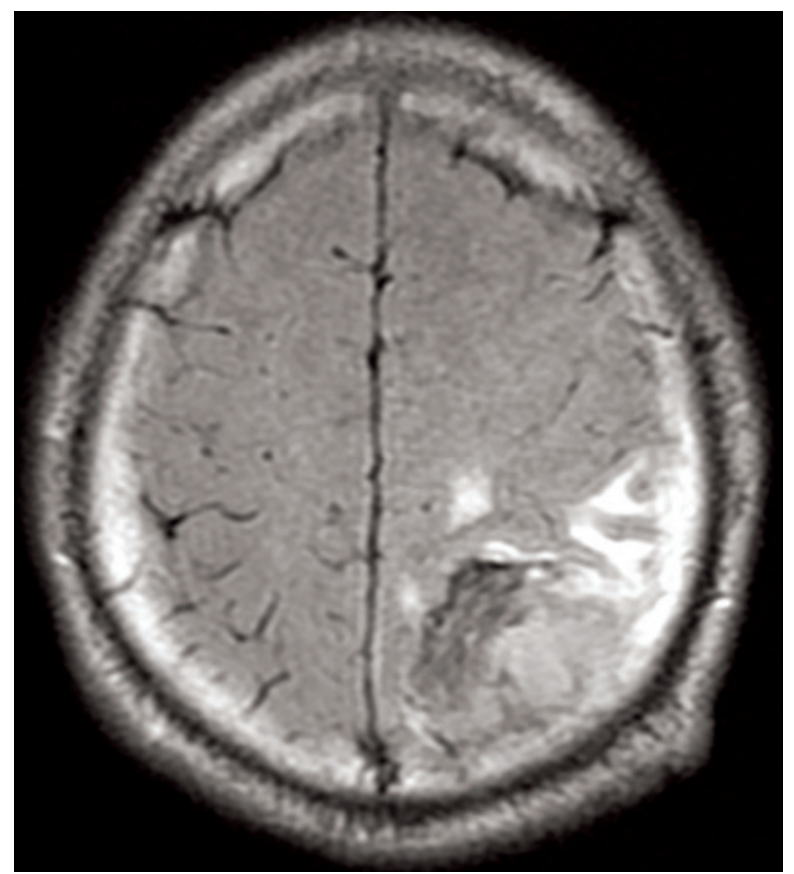

Figure 6: Mixed signal intensity on T2 FLAIR. 
entire chest region, over the entire back "bathing trunk", both upper and lower limbs and variously sized satellite lesions over the entire body.

A formal definition of neurocutaneous melanosis was not published until 1972 and then again in 1991, where it was defined as having meningeal melanosis in patients with large or multiple (>3) congenital nevi without evidence of cutaneous melanoma. The following

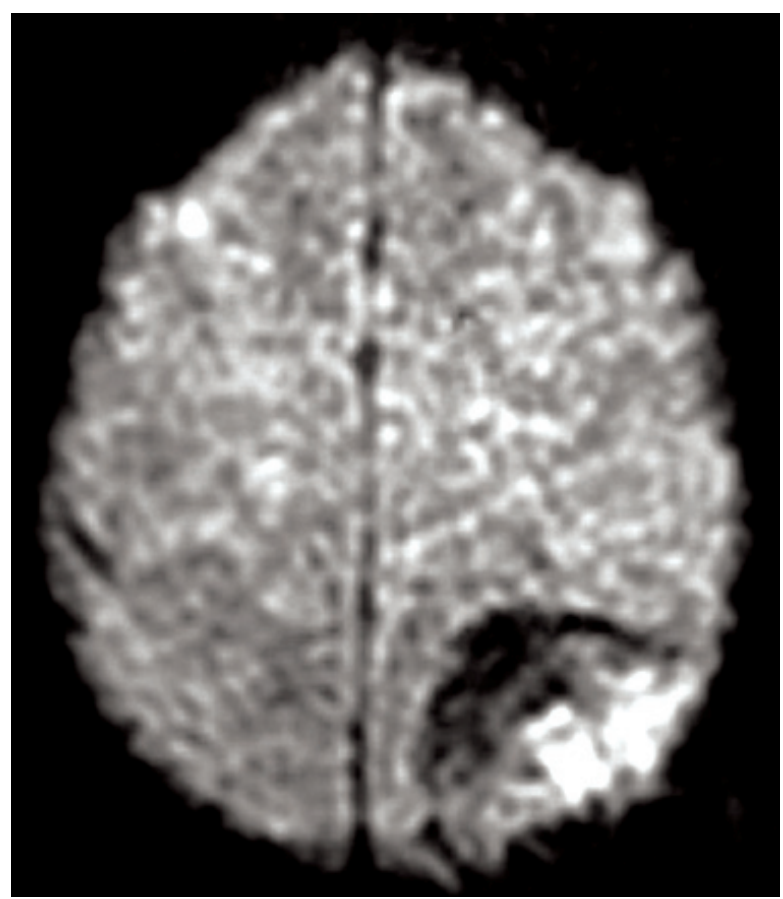

Figure 7: Mixed signal intensity on DWI.

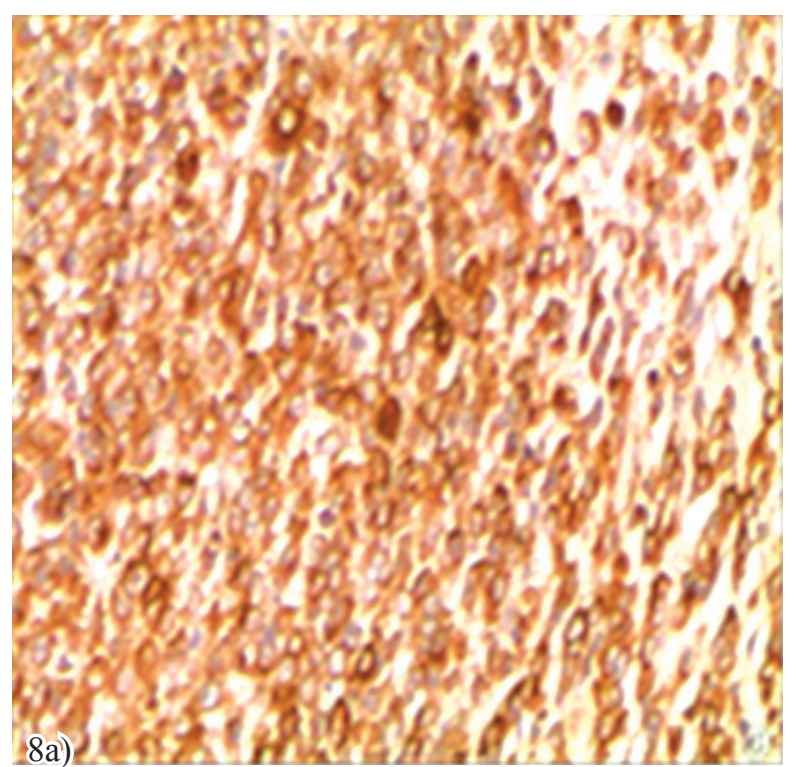

diagnostic criteria have been proposed by Kadonaga and Frieden:

(1) Large or multiple congenital nevi in association with meningeal melanosis or melanoma;

(2) No evidence of cutaneous melanoma except in patients in whom the examined areas of the meningeal lesions are histologically benign; and

(3) No evidence of meningeal melanoma except in patients in whom the examined areas of the cutaneous lesions are histologically benign.

Though definitive diagnosis of neurocutaneous melanosis requires histological confirmation of leptomeningeal melanocytic deposits, a provisional diagnosis can be established in patients if the above criteria are met $^{2}$. Six percent to $11 \%$ of patients with giant congenital nevi develop symptomatic neurocutaneous melanosis, and those with nevi involving the head, posterior neck, or paravertebral area are at the greatest risk. These patients are also at risk for developing leptomeningeal melanoma and cutaneous melanoma. The development of benign melanocytic proliferation of the leptomeninges has a poor prognosis, and neither radiation therapy nor chemotherapy improves the patient outcome. Patients with neurocutaneous melanosis are reported to develop malignant melanoma in approximately $40 \%-60 \%$ of case. Faillace et al have suggested that malignant transformation is heralded by development of intraparenchymal invasion or intracranial or intraspinal masses ${ }^{3}$. The majority of patients die within 3 years from benign overgrowth

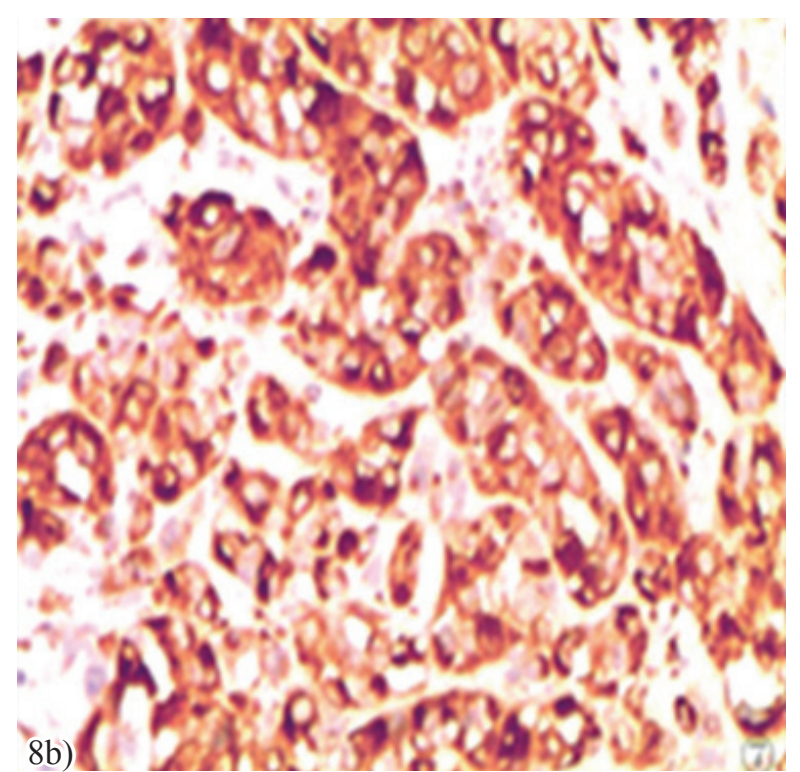

Figure 8: a) and b) Figure shows the immunohistochemistry of malignant melanoma specific HMB45 and Melan-A sensitive tumor marker expression respectively. 
of melanocytic cells or development of malignant melanoma ${ }^{3}$. In our patient, neurological symptoms were manifested only in third decade of life, which is rare in a case of neurocutaneous melanosis presented with giant melanocytic nevi.

NCM is known to be associated with the other Neurocutaneous syndromes, such as Sturge-Weber syndrome and von Recklinghausen's disease. Associations with Dandy-Walker complex, spinal lipomas, and arachnoid cysts have also been ${ }^{8}$.

The primary malignant melanoma found in central nervous system accounts for approximately 1\% of all the cases of melanomas. They are usually found as leptomeningeal melanoma, and primary cerebellopontine angle and sellar melanocytic tumors are also reported. As primary intracranial melanoma are rare, a metastatic one was first to be considered'. His metastatic evaluation included clinical examination for intraocular and dermal primary melanoma and was found negative.

The imaging clue to the diagnosis of leptomeningeal melanosis or parenchymal melanin deposits on
T1sequence shortening on MRI. This is often ascribed to the paramagnetic metal scavenging of melanoma cells resulting in a spontaneous high signal of melanin on $\mathrm{T} 1$ sequence. Other authors attribute $\mathrm{T} 1$ sequenceshortening to paramagnetic free radicals known to occur in melanin ${ }^{10}$. In our patient we noticed $\mathrm{T} 1$ sequence shortening along the brain parenchyma.

\section{CONCLUSION}

Neurocutaneous melanosis is a rare congenital neurocutaneous disorder characterized by congenital neurocutaneous nevi in association with leptomeningeal melanosis. In our case,patient was not evaluated for neurocutaneous melanosis in childhood. The onset of neurological symptoms only in third decade of life in our patientillustrates the importance of early multidisciplinary evaluation in order to detect neurological symptoms and/or melanoma in patient with neurocutaneous melanosis presented with giant melanocytic nevi.

\section{ACKNOWLEDGEMENTS}

We are grateful to our patient's parents for giving us permission to publish this case with pictures.

\section{REFERENCES}

1. McClelland S, 3rd, Charnas LR, SantaCruz KS, Garner HP, Lam CH. Progressive brainstem compression in an infant with neurocutaneous melanosis and DandyWalker complex following ventriculoperitoneal shunt placement for hydrocephalus. Case report. Journal of neurosurgery. 2007;107(6 Suppl):500-3.

2. Ha SK, Lee JM, Kim ER, Hwang H, Lee HT. A case of congenital neurocutaneous melanosis. Korean Journal of Pediatrics 2006;49(2).

3. Gouveia P, Bastos LP, Lopes dSS, Rebelo O, Dias A. Manifestation of Neurocutaneous Melanosis in an adult age European Society of Radiology. 2007.

4. Acosta FL, Jr., Binder DK, Barkovich AJ, Frieden IJ, Gupta N. Neurocutaneous melanosis presenting with hydrocephalus. Case report and review of the literature. Journal of neurosurgery. 2005;102(1 Suppl):96-100.

5. Viana AC, Gontijo B, Bittencourt FV. Giant congenital melanocytic nevus. Anais brasileiros de dermatologia. 2013;88(6):863-78.

6. Ramaswamy V, Delaney $H$, Haque S, Marghoob A, Khakoo Y. Spectrum of central nervous system

abnormalities in neurocutaneous melanocytosis. Developmental medicine and child neurology. 2012;54(6):563-8.

7. Smith $A B$, Rushing EJ, Smirniotopoulos JG. Pigmented lesions of the central nervous system: radiologic-pathologic correlation. Radiographics : a review publication of the Radiological Society of North America, Inc. 2009;29(5):1503-24.

8. Rai S, Kalakoti P, Syed MA, Thacker PJ, Jain R, Kalra G. An unusual case of congenital melanocytic nevus presenting as neurocutaneous melanoma coexisting with Tuberous Sclerosis complex: A case report. Journal of medical case reports. 2011;5:267.

9. Chen X, Zhimao J, Yuzhuo H, Peng G, Wu X, Feng $Y$, et al. Congenital melanocytic nevi with primary cerebral melanoma: a rarity. APSP journal of case reports. 2013;4(3):43.

10. Vanzieleghem BD, Lemmerling MM, Van Coster RN. Neurocutaneous melanosis presenting with intracranial amelanotic melanoma. AJNR American journal of neuroradiology. 1999;20(3):457-60. 